\title{
Surveillance study of species distribution, antifungal susceptibility and mortality of nosocomial candidemia in a tertiary care hospital in China
}

Chun-fang Ma ${ }^{1}$, Fang-qiu Li ${ }^{1,2^{*}}$, Li-ning Shi ${ }^{1}$, Yu-an Hu${ }^{1}$, Ying Wang ${ }^{1}$, Mei Huang ${ }^{1}$ and Qian-qian Kong ${ }^{1}$

\begin{abstract}
Background: Bloodstream infections due to Candida species cause significant morbidity and mortality, and the epidemiology of Candida infection is changing. Surveillance for candidemia is necessary to detect trends in species distribution and antifungal resistance.

Methods: The medical and electronic records of all patients who had candidemia at the authors' hospital from 2009 to 2011 were reviewed for demographic data and clinical information, including the infecting Candida species, resistance to antifungals and survival, and the presence of risk factors associated with candidemia.

Results: A total of 133 distinct episodes of candidemia were identified over the study period. The annual incidence of candidemia ranged between 0.71 and 0.85 cases/1000 hospital discharges. The most frequent Candida species were C. tropicalis (28.6\%), followed by C. albicans (23.3\%) and C. parapsilosis (19.5\%). The rates of susceptibility to antifungal agents were as followed: voriconazole (97.8\%), itraconazole (69.5\%), fluconazole (46.1\%), ketoconazole (38.9\%). Out of 131 evaluable patients, 34 (26.0\%) died within 30 days from the onset of candidemia. C. tropicalis candidemia was associated with the highest mortality rate (44.7\%). Regarding the crude mortality in the different units, patients in Hemato-Oncology ward had the highest mortality rate (66.7\%), followed by patients in cardiovascular wards and ICU (57.1\% and $25.6 \%$, respectively). Predictors of 30-day mortality were identified by uni- and multivariate analyses. Complicated abdominal surgery, presence of central venous catheter (CVC), neutropenia, candidemia due to $C$. tropicalis and poor treatment with fluconazole were significantly associated with the 30-day mortality. Presence of $\mathrm{CVC}$ (odds ratio[OR] $=4.177 ; 95 \%$ confidence interval $[\mathrm{Cl}]=1.698$ to $10.278 ; P=0.002$ ) was the only independent predictor for mortality in the multivariate analysis.
\end{abstract}

Conclusion: This report provides baseline data for future epidemiological and susceptibility studies and for the mortality rates associated with candidemia in our hospital. The knowledge of the local epidemiological trends in Candida species isolated in blood cultures is important to guide therapeutic choices.

Keywords: Nosocomial candidemia, Antifungal susceptibility, Mortality, Risk factors

\footnotetext{
*Correspondence: njlifq@163.com

'Laboratory of Molecular Biology, Institute of Medical Laboratory Sciences,

Jinling Hospital, School of Medicine, Nanjing University, Nanjing 210002, PR

China

${ }^{2}$ Laboratory of Molecular Biology, Institute of Medical Laboratory Sciences,

Jinling Hospital, 305 East Zhongshan Road, Nanjing 210002, P. R. China
} 


\section{Background}

Candida spp. is one of the most frequent pathogens isolated in bloodstream infections (BSI), and is associated with significant morbidity and mortality [1,2]. During the past two decades, the incidence of candidemia has been doubled and Candida spp. currently ranks as the fourth and the seventh most common bloodstream pathogen in North American and European studies [3,4]. The reasons for the increasing candidemia rates include improved detection as well as increase in patientpopulation at risk, such as invasive procedures and devices, broad-spectrum antimicrobial agents, advanced life-support and aggressive chemotherapy are more extensively used [5].

Candidemia have been associated with high crude and attributable mortality rates, especially among critically ill patients. The crude mortality rate of these infections is $40 \%-75 \%$ and the attributable mortality of candidemia has been estimated at 25\%-38\% [6,7]. In addition, an increase of 30 days in the length of hospital stay among patients surviving these infections has been demonstrated [8]. The economic impact of these infections is also important: candidemia has been associated with increased costs of care and prolonged hospitalization [9].

Historically, Candida albicans is the most common cause of candidemia worldwide. However, in recent years, some studies have reported an increase of candidemia due to non-albicans Candida species, with the threat of increased mortality and antifungal drug resistance [10,11]. The intrinsic and emerging resistance to azoles actually represents a major challenge for empirical therapeutic and prophylactic strategies [12].

The epidemiology of candidemia shows a wide variation among different countries. For example, an increasing incidence of candidemia in Iceland was reported for the period between 2000 and 2011 [13], but the similarity was not observed in Switzerland, where a national surveillance study showed that the incidence of candidemia had remained unchanged during the period of 1991 to 2000 [4]. Despite the epidemiology of candidemia has been studied extensively in the United States, Europe, and some countries in South America, very few studies have addressed these issues in China, where the differences about the demographic characteristics, health care practices, patterns using blood cultures, and antibiotic usage as well as the resistance situation do exist compared to other countries. In the present study, a three-year retrospective analysis was conducted to evaluate the incidence, species distribution, the associated resistance patterns of Candida species for the contemporary azole antifungal agents, outcome of candidema BSI and risk factors for mortality in patients with candidemia in Jinling hospital, Nanjing, China.

\section{Methods}

\section{Collection of patients}

In our previous study, we developed two enzyme-linked immunosorbent assays to detect specific antibodies against Candida Eno and Fba1, and investigated the diagnostic value for invasive Candida infections by analyzing sera from patients with candidemia [14]. The purpose of current study was to investigate the state of Candida invasive infection. We conducted a retrospective observational study of computerized laboratory records of positive blood cultures at Jinling Hospital (Nanjing, China), a 1,800 beds tertiary care hospital with about 55,000 admissions per year from January 2009 to December 2011. The target population consisted of hospitalized patients presenting risk factors for candidemia, especially patients receiving broad-spectrum antibiotics, immunosuppressive therapy, parenteral nutrition, abdominal/thoracic surgery and hematopoietic transplantation, or those patients who had a long intensive care unit stay or acute renal failure. An episode of candidemia was defined as the isolation of a Candida species from blood culture in a patient with temporally related clinical signs and symptoms, such as fever, chills, or hypotension. For each patient, only the first episode of candidemia was included. Patients whose cultures grew $>1$ species of Candida were excluded from the analysis and all data were collected by using the electronic medical records. The following data were retrospectively collected: basic demographics, underlying disease, the specific fungal pathogen and species, resistance to antifungals and survival. The presence of risk factors associated with candidemia were also collected: systemic corticosteroid treatment (a dose equivalent to prednisone $10 \mathrm{mg} / \mathrm{d}$ for at least 14 days), neutropenia (absolute neutrophil count $<500$ cells $/ \mu \mathrm{l}$ ), complicated abdominal surgery (severe acute pancreatitis, and complex ventral hernia), acute renal failure (sudden and often temporary loss of kidney function with nitrogen waste retention and hypourocrinia), indwelling central vascular catheter, intensive care unit hospitalization or mechanical ventilation. Early mortality was defined as death within seven days and late mortality as death between seven and 30 days [15]. The study protocol was approved by the Medical Ethics Committee of Jinling hospital.

\section{Organism identification}

A volume of $3 \mathrm{ml}$ blood for children and $5 \sim 10 \mathrm{ml}$ blood for adults was collected aseptically by skin venipuncture taken from different parts of each patient. The blood was inoculated into both BacT/AlerT 3D aerobic and anaerobic vials (Becton Dickinson). All positive cultures were manually sampled and inoculated on CHROMagar Candida medium (CHROMagar Company, Paris France) to ensure viability and purity. An aliquot was Gram-stained for preliminary identification of the microorganism. Identi- 
fication of all species was confirmed with the Vitek-2 system (bioMerieux, France).

\section{Agar diffusion method}

The growth inhibition zone and the category (resistant, intermediate and susceptible) were determined for fluconazole (FLC), itraconazole (ITC), ketoconazole (KTC), voriconazole (VOR), using a commercial agar diffusion test with Neo-Sensitabs ${ }^{\bullet}$ tablets (Rosco, Denmark). NeoSensitabs ${ }^{\circ}$ sensitivity testing is a standardized agar diffusion method which includes antifungal agents in tablets of $9 \mathrm{~mm}$ of diameter with FLC $(25 \mu \mathrm{g})$, ITC $(8 \mu \mathrm{g})$, KTC $(15 \mu \mathrm{g})$ and VOR $(1 \mu \mathrm{g})$. Neo-Sensitabs ${ }^{\circ}$ assay was performed according to the manufacturer's instructions [16]. Inocula contained $5 \times 10^{5}$ cells $/ \mathrm{ml}$ and were prepared from an overnight subculture on Sabouraud glucose agar (Difco, USA), getting suspensions corresponding to McFarland 0.5 standard and then diluted (1:1) with sterile saline solutions. For $C$. krusei isolates inocula were equivalent to McFarland 0.5 standard, diluted 1:10 in saline solutions. Two milliliters of each inoculum suspensions were poured onto the agar surface and later removing the liquid in excess. Opened plates were dried at $35^{\circ} \mathrm{C}$ for $15 \mathrm{~min}$ before tablets will be placed on the agar surface.

Shadomy modified medium was used (Yeast Nitrogen Base, asparagine and glucose) with phosphate buffer to pH 7 and containing chloramphenicol to avoid bacterial contamination. Media was heated at $100^{\circ} \mathrm{C}$ during $15 \mathrm{~min}$, and then cooled to $60^{\circ} \mathrm{C}$ before pouring the medium in Petri dishes $(12 \mathrm{~cm} \times 12 \mathrm{~cm})$. A pre-diffusion drying was made at $37^{\circ} \mathrm{C}$ during $15 \mathrm{~min}$. After an incubation period of 20-24 h, the inhibition diameter zones were read in $\mathrm{mm}$ with a caliper (Mitutoyo, Japan). Zone diameters were measured to the nearest whole millimeter at a point in which there was a prominent reduction of growth $(80 \%$ for azoles). Depending on the diameter of the inhibition zone, Candida strains were classified as susceptible (S), intermediate (I) or resistant (R) to tested antifungals. The basic criterion according to manufacturers instructions was as follows: FLC ( $\mathrm{S} \geq 19 \mathrm{~mm}, \mathrm{I} 18 \sim 15 \mathrm{~mm}, \mathrm{R} \leq 14 \mathrm{~mm}$ ); ITC ( $\mathrm{S} \geq 15 \mathrm{~mm}$, I14 10 mm, R $<10 \mathrm{~mm}$ ); KTC $(\mathrm{S} \geq 28 \mathrm{~mm}$, I27 $21 \mathrm{~mm}, \mathrm{R} \leq 20 \mathrm{~mm})$; VOR $(\mathrm{S} \geq 17 \mathrm{~mm}, \mathrm{I} 16 \sim 14 \mathrm{~mm}$, $\mathrm{R} \leq 13 \mathrm{~mm}$ ).

\section{Statistical analyses}

In the assessment of predictors of poor outcome, we compared patients who survived until day 30 after the incident candidemia with those who died. Univariate analysis was perforemd using Fisher exact test or Chisquared test for categorical variables. All variables with $P$-value $<0.1$ by univariate analysis were entered in a logistic regression model. Kaplan-Meier curves were constructed to assess survival, and curve comparisons were performed by the Log Rank test. All tests were two-tailed and a level of significance of $P<0.05$ was considered statistically significant. Statistical analysis was performed using SPSS 18.0 for Windows (SPSS, Chicago, IL, USA).

\section{Results}

A total of 133 distinct episodes of candidemia (130 adults and 3 children $<15$ years of age) were identified during the study period. Most candidemia was reported in adults (97.7\%). More candidemia occurred among males (68.4\%) than females, mainly in those over 60 years of age (Table 1). The mean annual incidence of candidaemia was 0.77 per 1000 admission. The incidence of candidemia increased from 0.71 in 2009 to 0.85 episodes/1000 admission in 2011. But the change in incidence over time did not reach statistical significance. The demographic and clinical characteristics of the patients are summarized in Table 1. Most patients had one or more comorbidity at the time of the diagnosis of candidaemia. The most common underlying conditions documented prior to candidemia were intestinal fistula/abdominal infection (55.7\%), respiratory dysfunction (12.8\%), and gastrointestinal pathology (10.2\%).

The percentages of the three most common Candida spp. isolated were as follows: C. tropicalis (28.6\%), C. albicans (23.3\%) and C. parapsilosis (19.5\%) (Table 1). The causative organism varied according to age of patients and their underlying diseases. With increasing age, a reduction in the percentage of C. albicans (from $33.3 \%$ to $23 \%$ ) and C. tropicalis (from $33.3 \%$ to $27.9 \%$ ) and a significant increase of C. parapsilosis (from 0 to 21.3\%) was observed. Non-albicans Candida species were isolated with the highest frequency from patients with respiratory dysfunction (82.4\%) and haematological malignancies (83.3\%), in which C. tropicalis was the predominant species $(64.7 \%$ and $83.3 \%$, respectively).

The distribution of isolated Candida species is shown in Figure 1. Globally, the high rates of candidemia infection are not limited to ICU but also occurred on general wards. Out of 133 evaluable patients, 51 (38.3\%) were hospitalized in ward of general surgery, this proportion being a small amount higher than that which arose from patients in ICU (23.3\%). The distribution of Candida albicans and non-albicans Candida species differed according to the type of patient population and risk factors, as shown in Figure 1 . In patients with hematologic malignancies, $C$. albicans accounted for $16.7 \%$ of the cases and C. tropicalis for $83.3 \%$; In ICU C. albicans was isolated in $17.9 \%$ of the cases, C. tropicalis in $30.8 \%$ and C. parapsilosis in $20.5 \%$; on the other hand in oncology C. albicans was isolated in $50 \%$ of the cases.

Table 2 summarizes the results of in vitro susceptibility testing of bloodstream isolates of Candida in the study. Consistent with previous reports $[17,18]$, vorconazole 
Table 1 Patient characteristics and incidence (episode/1000 admission)

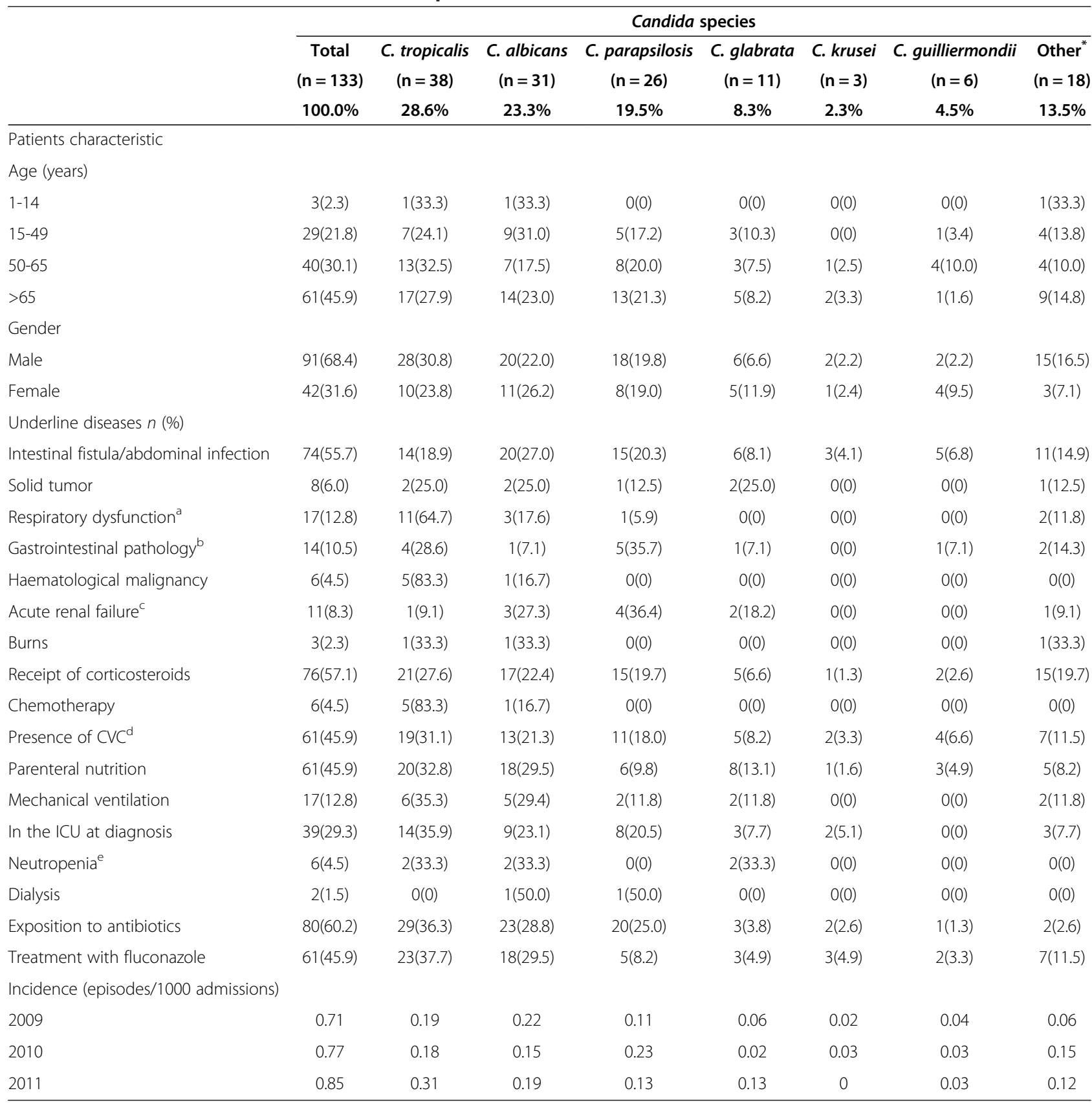

"Others include: C. pelliculosa(8), C. famata(7) and C. haemulonii(2).

ancludes the following diseases: pneumonia, chronic obstructive pulmonary disease, and acute respiratory distress syndrome.

${ }^{b}$ Includes the following diseases: cholecystitis, pancreatitis, peritonitis, and hepatitis.

${ }^{\mathrm{C}}$ Acute renal failure was defined as sudden and often temporary loss of kidney function with nitrogen waste retention and hypourocrinia.

${ }^{\mathrm{d}}$ CVC central venous catheter.

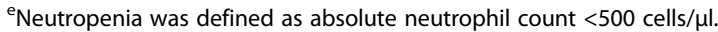

demonstrated excellent in vitro potency against Candida species, nearly all strains (97.8\%) were susceptible to VOR. With regard to KTC, the rate of resistant is particularly high in Candida spp. (61.9\%), almost all isolates were resistant to KTC. ITC resistance was observed for 30.5\% of all Candida isolates evaluated and was also highest among C. glabrata isolates (90.9\% resistance). Most strains (53.9\%) became resistant to FLC, only $8 \mathrm{C}$. albicans BSI isolates (25.8\%) collected during the 3-year surveillance program were reported as resistant. Decreased susceptibility to FLC was mostly seen with C. glabrata (90.9\% resistance) and C. tropicalis (68.4\% resistance). The percentages of yeast isolates with resistance and decreased susceptibility to FLC were similar during the three phases. 


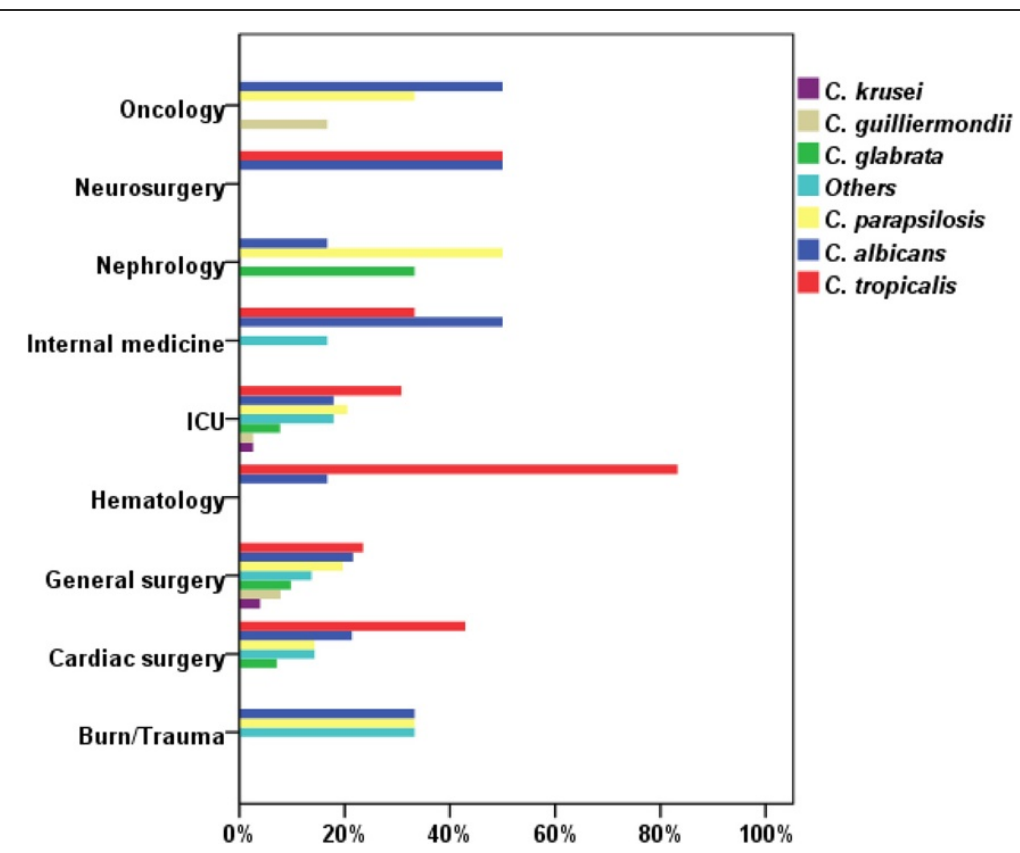

Figure 1 Distribution of the Candida species according to underlying pathology/medical care (n).

Outcome at day 30 was available for 131 episodes; patients in the remaining 2 episodes were lost to follow up. The crude mortality rate was $26.0 \%$; Seven patients died within 48 hours of obtaining the blood culture, the time by which positive blood culture reports became available. This overall mortality rate varied according to the patients with different species of Candida, as well as the underlying disease or condition, the highest mortality rate was observed for C. tropicalis fungemia (44.7\%). Haematological malignancy (66.7\%), cardiovascular disease (57.1\%), and intensive care in adults (25.6\%) were associated more frequently with death during the study period.

Univariate predictors of poor outcome in candidemia is shown in Table 3. For candidemic patients, the variables associated with 30-day mortality were as follows: complicated abdominal surgery, presence of $\mathrm{CVC}$, neu- tropenia, candidemia due to C. tropicalis. Major factor associated with 30-day survival was receipt of fluconazole as primary treatment for candidemia. Because neutropenia was associated with poor outcome and this variable was available in only 6 of the 131 patients, this variable was not included in the multiply logistic regression model. As shown in Table 4, predictors of 30-day mortality by multivariate analysis among patients with candidemia were presence of CVC (OR 4.177; 95\% CI 1.69810.278; $P=0.002$ ).

\section{Discussion}

In recent years, some studies have reported that the incidence of candedimia is increasing in many hospitals around the world. Our data show that in our hospital the incidence of candidemia has increased steadily in the

Table 2 Antifungal susceptibility of Candida bloodstream isolates in patients during the study period

\begin{tabular}{|c|c|c|c|c|c|c|c|c|}
\hline \multirow[b]{2}{*}{ Species } & \multicolumn{2}{|l|}{ Fluconazole } & \multicolumn{2}{|l|}{ Itraconazole } & \multicolumn{2}{|l|}{ Ketoconazole } & \multicolumn{2}{|l|}{ Voriconazole } \\
\hline & No. of isolates tested & $\% \mathrm{~S}^{\#}$ & No. of isolates tested & $\% \mathrm{~S}$ & No. of isolates tested & $\% \mathrm{~S}$ & No. of isolates tested & $\% \mathrm{~S}$ \\
\hline C. albicans & 31 & $23(74.2)$ & 31 & 29 (93.6) & 6 & $3(50.0)$ & 22 & $22(100.0)$ \\
\hline C. parapsilosis & 26 & $15(57.7)$ & 26 & $25(96.2)$ & 2 & $1(50.0)$ & 22 & $22(100.0)$ \\
\hline C. glabrata & 11 & $1(9.1)$ & 11 & $1(9.1)$ & 1 & $0(0)$ & 5 & $5(100.0)$ \\
\hline C. tropicalis & 38 & 12 (31.6) & 38 & $25(65.8)$ & 5 & $0(0)$ & 23 & $21(91.3)$ \\
\hline C. krusei & 3 & $0(0)$ & 3 & $1(33.3)$ & 1 & $0(0)$ & 3 & $3(100.0)$ \\
\hline C. guilliermondii & 6 & $4(66.7)$ & 6 & $5(83.3)$ & 0 & $0(0)$ & 5 & $5(100.0)$ \\
\hline Other ${ }^{*}$ & 16 & $4(25.0)$ & 16 & $5(31.3)$ & 3 & $3(100.0)$ & 12 & $12(100.0)$ \\
\hline Total & 128 & $59(46.1)$ & 131 & $91(69.5)^{*}$ & 18 & $7(38.9)$ & 92 & $90(97.8)$ \\
\hline
\end{tabular}

*; Others include: C. pelliculosa, C. famata and C. haemuloni.

\#; Abbreviations: $S$ susceptible. 
Table 3 Factors associated with 30-day mortality by univariate analysis in candidemic patients

\begin{tabular}{|c|c|c|c|}
\hline \multirow[b]{2}{*}{ Variable } & \multicolumn{2}{|c|}{ 30-day outcome } & \multirow[b]{2}{*}{$P$-value } \\
\hline & $\begin{array}{c}\text { Survived } \\
\mathrm{N}=97\end{array}$ & $\begin{array}{c}\text { Died } \\
\mathrm{N}=34\end{array}$ & \\
\hline Median age, years (range) & $61(0-88)$ & $69(0-73)$ & 0.58 \\
\hline Gender (male:female) & $68: 29$ & 23:11 & 0.889 \\
\hline In the ICU at diagnosis, $n(\%)$ & $30(30.9)$ & $9(26.5)$ & 0.457 \\
\hline Mechanical ventilation, $n$ (\%) & $10(10.3)$ & $7(20.6)$ & 0.109 \\
\hline Parenteral nutrition, $n$ (\%) & $43(44.3)$ & $18(52.9)$ & 0.952 \\
\hline Complicated abdominal surgery, $n$ (\%) & $21(21.6)$ & $14(41.2)$ & 0.019 \\
\hline Presence of CVC, $n(\%)$ & $34(35.1)$ & $27(79.4)$ & $<0.001$ \\
\hline Receipt of corticosteroids, n (\%) & $53(54.6)$ & $23(67.6)$ & 0.397 \\
\hline Neutropenia, $n(\%)$ & $2(2.1)$ & $4(11.8)$ & 0.015 \\
\hline \multicolumn{4}{|l|}{ Species, $n$ (\%) } \\
\hline C. albicans & $25(25.8)$ & $6(17.6)$ & 0.508 \\
\hline C. tropicalis & $21(21.6)$ & $17(50.0)$ & 0.002 \\
\hline C. parapsilosis & 19 (19.6) & $5(14.7)$ & 0.444 \\
\hline C. glabrata & $8(8.2)$ & $3(8.8)$ & 0.928 \\
\hline C. krusei & $2(2.1)$ & $1(2.9)$ & 0.575 \\
\hline Treatment with antibiotics, $n(\%)$ & $57(58.8)$ & $23(67.6)$ & 0.600 \\
\hline Treatment with fluconazole, $n(\%)$ & $51(52.6)$ & $10(29.4)$ & 0.019 \\
\hline
\end{tabular}

ICU intensive care unit, CVC central venous catheter.

past three years in parallel with medical technological advances. The incidence is somewhat higher than that reported for centers in Denmark (0.41 case per 1000 admissions) [19], Israel (0.50 case per 1000 admissions) [20], China (0.53 cases per 1000 admissions) [21], and much lower than that reported in Brazil (1.87 cases per 1000 admissions) [22]. The differences in candidemia rates between countries may reflect differences in demographic characteristics, variations in health care practice, patterns using blood cultures and long duration of antibacterial usage as well as the resistance situation.

Neonates and infants have historically been populations with some of the highest rates of candidemia [23-29]. In our study, BSI by candida species occurred more frequently among males, mainly in those over 65 years old. The reasons for the shift in burden from neonates to

Table 4 Factors associated with 30-day mortality by multivariate analysis

\begin{tabular}{lccc}
\hline Variable & Odds ratio & $\begin{array}{c}\text { 95\% Confidence } \\
\text { interval }\end{array}$ & P-value \\
\hline $\begin{array}{l}\text { Complicated abdominal } \\
\text { surgery }\end{array}$ & 1.678 & $0.802-3.510$ & 0.169 \\
Presence of CVC & 4.177 & $1.698-310.278$ & 0.002 \\
C. tropicalis & 1.705 & $0.809-33.593$ & 0.161 \\
Treatment with fluconazole & 0.523 & $0.247-31.110$ & 0.091 \\
\hline
\end{tabular}

CVC central venous catheter. adults are likely multifactorial; some contribution may be due to changes in the prevalence of risk factors in the adult population. Increases in common risk factors such as ICU admission [30,31], or numbers of patients receiving immunosuppressive therapies [32] may have resulted in increase in the overall pool of patients at high risk for candidemia. The difference in the distribution of cases between genders could be related to the predominant presence of male patients in units where the use of invasive devices, broad-spectrum antibiotics, extensive surgical procedures, or advanced life support is frequently used.

Over the past 20 years, a shift towards non-albicans Candida species has been reported previously from the USA, Europe and Australia, although the precise pattern of causative species varies across countries [5]. The findings from our surveillance are partially supportive of these reports. We observed a significant predominance of non-albicans Candida species (76.7\%), with C. tropicalis being the most common isolate $(28.6 \%)$, followed by $C$. albicans (23.3\%), C. parapsilosis (19.5\%) and C. glabrata (8.3\%). Traditionally, C. tropicalis has been the second most common Candida species recovered from blood [33]. Similarly, as previously reported at a hospital in China, C. albicans (57.8\%) continued to play a dominant role in candidemia, followed by C. tropicalis (12.8\%) [34]. In our report, $C$. tropicalis surpassed the other Candida species to become the most common species, we recognize that this finding may simply be a result of the small sample size, and the further studies with larger sample size are needed to verify it. It is worth mentioning that eight C. pelliculosa, seven C. famata and two C. haemulonii isolates were recovered during the study period, suggesting the diversity of the etiologic agents. ARTEMIS DISK Global antifungal surveillance also found an increase of the involvement of less-common Candida species during the 10.5-year study period and showed that species identification is important to diagnosis and surveillance [35].

As already reported [36]. non-albicans Candida species were predominant in patients with haematological malignancies (83.3\%), a finding in accordance with the global results of the ECMM survey [7]; moreover, $C$. tropicalis was frequently isolated in this group of patients, the prevalence of $C$. tropicalis among patients with haematological malignancies is consistent with previous reports [19].

The antifungal susceptibility patterns revealed that voriconazole has excellent in vitro activity overall against Candida species. Successful salvage therapy with voriconazole for the treatment of candidemia in patients intolerant or refractory to other antifungal agents has been reported [37]. Some studies showed that voriconazole may be a suitable agent for salvage therapy of invasive candidiasis, even in the setting of previous azole expo- 
sure and C. krusei infection [36,38]. Most publications on antifungal resistance over the past 10 years have been concerned with resistance to triazole antifungals, especially fluconazole, itraconazole, and ketoconazole. For ketoconazole, it showed the exsitence of a sensitivity of $38.1 \%$ of strains studied, lower than the other three azoles. Ketoconazole resistance was observed in 50\% of C. albicans and C. parapsilosis, respectively. Itraconazole resistance was observed for $30.5 \%$ of all Candida species and was also highest among C. glabrata (90.9\% resistance), this sensitivity decline was similar to that previously published in Iowa Organisms Study [39]. In recent years, resistance to azole antifungal agents among Candida spp. is still uncommon. In Iceland, $97.3 \%$ of the Candida spp. isolates tested were susceptible to fluconazole [13]. A similar data has been reported from North India [40]. In contrast to other reports, antifungal resistance was a notable finding in our study and was mainly restricted to fluconazole. Our proportion of fluconazoleresistant isolates $(53.9 \%)$ was higher than the rates observed with spain (9.8\%) [41]. We propose two potential reasons, first, the number of isolates in this study is still not high enough, therefore the rate of azole resistance is higher than clinical reports. Second, the increasing use of fluconazole as antifungal therapy leads to a reduction in susceptibility and the appearance of resistant strains. Some reportes have demonstrated that candidemia due to non-albicans species increased and that was apparently correlated with an increasing use of azoles for prophylaxis or empirical treatment [42], in our study, almost all patients received empirical therapy, 65(45.9\%) patients were treated with fluconazole, followed by voriconazole (18.8\%), and caspofungin (11.3\%). For the latter reason, it would be convenient to carry out antifungal susceptibility studies in order to establish the in vitro activities of antifungal agents against local isolates and also to detect shifts toward resistance as early as possible. When analyzed by species, apart from the intrinsically fluconazole-resistant species (C. krusei), the highest rate of resistance to fluconazole was for $C$. glabrata and C. tropicalis (more than 50\%), which was consistent with other studies in whom the greatest resistance to fluconazole also showed C. glabrata (36\%) [41]. Furthermore, discrepancies in vitro susceptibility to FLC rate of different strains studied in this single-center study could be associated to differences in species distribution of isolates tested in this study.

In the present study we decided to analyze prognostic factors in patients with candidemia, we found that complicated abdominal surgery, presence of CVC, neutropenia, candidemia due to $C$. tropicalis, poor treatment with fluconazole were predictors of mortality in the univariate analysis. Of all the variables significantly associated with mortality in the univariate analysis, presence of CVC was the only predictive factor of mortality in the multivariate analysis. This is in agreement with the findings of a hospital population-based surveillance study [43]. In a study of pediatric candidemia, Candidemiaassociated mortality has been found to be $31-72 \%$ [44]. Similarly, the crude 30 -day mortality rate in our study was $26 \%$ for all patients with candidemia, which is in concordance with rates reported from Chinese tertiarycare centers, ranging from $26.4 \%$ to $33.3 \%$ [21,34]. A possible explanation for the relatively low mortality of nosocomial candidemia is the increase in empirical and/ or pre-emptive use of fluconazole for presumed invasive candidiasis. Furthermore, although the hospital is a tertiary care hospital it rarely has patients with solid organ or bone marrow transplants. Thus, on average the patients in our hospital are less sick than in other hospitals. In our study, C. tropicalis was the species associated with the highest mortality rate (50\%), besides being raised among the elderly, mortality is particularly high among patients with cancer $(66.7 \%$ for haematological malignancies) as reported previously $[33,45,46]$. Certainly the severity of the underlying medical conditions has greatly influenced the crude mortality rate in these patient populations.

\section{Conclusions}

This report shows that candidemia is a significant source of morbidity in Nanjing, with a substantial burden of disease, mortality, and likely high associated costs. We concede that there are several potential limitations to the present study. First, this is a single-center study. Our conclusions might be influenced by the local ecology, management practices, infections control policy, or our own susceptibility patterns. Second, our analysis was restricted to adult patients. Therefore, our conclusions cannot be extrapolated to pediatric populations. Finally, the results of the multivariate analyses might be influenced by the sample size and the number of variables included in the models. However, it would be of immense value to establish surveillance of candidemia to develop and evaluate prevention strategies and to monitor for changes in incidence and resistance.

\section{Competing interests \\ The authors declare that they have no competing interests.}

\section{Authors' contributions}

FQL conceived, coordinated and designed the research; CFM performed the experiment, contributed to the acquisition, analysis and interpretation of data and drafted the manuscript. LNS, YAH, YW and MH participated in Candida spp. identification; QQK participated in sample collection and data acquisition. All the authors have read and approved the final manuscript.

\section{Acknowledgements}

This work was supported financially by Jiangsu Science Foundation (BE2009673). 
Received: 6 January 2013 Accepted: 18 July 2013

Published: 22 July 2013

\section{Reference}

1. Tortorano AM, Dho G, Prigitano A, Breda G, Grancini A, Emmi V, Cavanna C, Marino G, Morero S, Ossi C: Invasive fungal infections in the intensive care unit: a multicentre, prospective, observational study in Italy (2006-2008). Mycoses 2012, 55(1):73-79.

2. Kourkoumpetis T, Manolakaki D, Velmahos G, Chang Y, Alam HB, De Moya MM, Sailhamer EA, Mylonakis E: Candida infection and colonization among non-trauma emergency surgery patients. Virulence 2010, 1(5):359.

3. Wisplinghoff $H$, Bischoff T, Tallent SM, Seifert $H$, Wenzel RP, Edmond MB: Nosocomial bloodstream infections in US hospitals: analysis of 24,179 cases from a prospective nationwide surveillance study. Clin Infect Dis 2004, 39(3):309-317.

4. Marchetti O, Bille J, Fluckiger U, Eggimann P, Ruef C, Garbino J, Calandra T, Glauser MP, Täuber MG, Pittet D: Epidemiology of candidemia in Swiss tertiary care hospitals: secular trends, 1991-2000. Clin Infect Dis 2004, 38(3):311-320.

5. Poikonen E, Lyytikäinen O, Anttila VJ, Koivula I, Lumio J, Kotilainen P, Syriälä H, Ruutu P: Secular trend in candidemia and the use of fluconazole in Finland, 2004-2007. BMC Infect Dis 2010, 10(1):312.

6. Zaragoza R, Pemán J: The diagnostic and therapeutic approach to fungal infections in critical care settings. Adv Sepsis 2008, 6:90-98.

7. Tortorano A, Peman J, Bernhardt H, Klingspor L, Kibbler C, Faure O, Biraghi E, Canton E, Zimmermann K, Seaton S: Epidemiology of candidaemia in Europe: results of 28-month European Confederation of Medical Mycology (ECMM) hospital-based surveillance study. Eur I Clin Microbiol Infect Dis 2004, 23(4):317-322.

8. De Rosa FG, Trecarichi EM, Montrucchio C, Losito AR, Raviolo S, Posteraro B, Corcione S, Di Giambenedetto S, Fossati L, Sanguinetti M: Mortality in patients with early-or late-onset candidaemia. J Antimicrob Chemother 2013, 68(4):927-935.

9. Olaechea P, Palomar M, León-Gil C, Alvarez-Lerma F, Jorda R, Nolla-Salas J, León-Regidor M: Economic impact of Candida colonization and Candida infection in the critically ill patient. Eur J Clin Microbiol Infect Dis 2004, 23(4):323-330

10. Oberoi JK, Wattal C, Goel N, Raveendran R, Datta S, Prasad K: Non-albicans Candida species in blood stream infections in a tertiary care hospital at New Delhi, India. Indian J Med Res 2012, 136(6):997.

11. Magill SS, Shields C, Sears CL, Choti M, Merz WG: Triazole cross-resistance among Candida spp.: case report, occurrence among bloodstream isolates, and implications for antifungal therapy. J Clin Microbiol 2006, 44(2):529-535.

12. Leroy O, Gangneux JP, Montravers P, Mira JP, Gouin F, Sollet JP, Carlet J, Reynes J, Rosenheim M, Regnier B: Epidemiology, management, and risk factors for death of invasive Candida infections in critical care: a multicenter, prospective, observational study in France (2005-2006). Crit Care Med 2009, 37(5):1612-1618.

13. Asmundsdottir $L R$, Erlendsdottir $H$, Gottfredsson M: Nationwide Study of Candidemia, Antifungal Use, and Antifungal Drug Resistance in Iceland, 2000 to 2011. J Clin Microbiol 2013, 51(3):841-848.

14. Li FQ, Ma CF, Shi LN, Lu JF, Wang Y, Huang M, Kong QQ: Diagnostic value of immunoglobulin $\mathrm{G}$ antibodies against Candida enolase and fructose-bisphosphate aldolase for candidemia. BMC Infect Dis 2013, 13:253.

15. Almirante B, Rodríguez D, Park BJ, Cuenca-Estrella M, Planes AM, Almela M, Mensa J, Sanchez F, Ayats J, Gimenez M: Epidemiology and predictors of mortality in cases of Candida bloodstream infection: results from population-based surveillance, Barcelona, Spain, from 2002 to 2003. J Clin Microbiol 2005, 43(4):1829-1835.

16. Colosi I, Faure O, Lebeau B, Colosi H, Junie M, Pelloux H: Comparison of two methods, E-test ${ }^{\oplus}$ and Neo-Sensitabs ${ }^{\oplus}$ tablet diffusion assay, for testing susceptibility of 93 Candida strains to amphotericin B, fluconazole, voriconazole, and caspofungin. Rev Sci Parasitol 2009, 10(1/2):112-122.

17. Diekema DJ, Messer SA, Hollis RJ, Boyken L, Tendolkar S, Kroeger J, Jones RN, Pfaller MA: A global evaluation of voriconazole activity tested against recent clinical isolates of $<\mathbf{i}>$ Candida $</ \mathbf{i}>$ spp. Diagn Microbiol Infect Dis 2009, 63(2):233-236.
18. Marcos-Arias C, Eraso E, Madariaga L, Carrillo-Muñoz AJ, Quindós G: In Vitro Activities of New Triazole Antifungal Agents, Posaconazole and Voriconazole, Against Oral Candida Isolates from Patients Suffering from Denture Stomatitis. Mycopathologia 2012, 173(1):35-46.

19. Arendrup MC, Bruun B, Christensen JJ, Fuursted K, Johansen HK, Kjældgaard P, Knudsen JD, Kristensen L, Møller J, Nielsen L: National surveillance of fungemia in Denmark (2004 to 2009). J Clin Microbiol 2011, 49(1):325-334.

20. Rennert G, Rennert H, Pitlik S, Finkelstein R, Kitzes-Cohen R: Epidemiology of candidemia-a nationwide survey in Israel. Infection 2000, 28(1):26-29.

21. Li D, Zhang $W$, Zheng $S, M a Z$, Zhang P, Liu Z: Surveillance study of candidemia in cancer patients in North China. Med Mycol 2013, 51(4):378-384.

22. Motta AL, Almeida GMD, Almeida Júnior JN, Burattini MN, Rossi F: Candidemia epidemiology and susceptibility profile in the largest Brazilian teaching hospital complex. Braz I Infect Dis 2010, 14(5):441-448.

23. Roilides E: Invasive candidiasis in neonates and children. Early Hum Dev 2011, 87:S75-S76.

24. Fridkin SK: Candidemia is costly — plain and simple. Clin Infect Dis 2005, 41(9):1240-1241.

25. Hajjeh RA, Sofair AN, Harrison LH, Lyon GM, Arthington-Skaggs BA, Mirza SA, Phelan M, Morgan J, Lee-Yang W, Ciblak MA: Incidence of bloodstream infections due to Candida species and in vitro susceptibilities of isolates collected from 1998 to 2000 in a population-based active surveillance program. J Clin Microbiol 2004, 42(4):1519-1527.

26. Pfaller M, Diekema D: Epidemiology of invasive candidiasis: a persistent public health problem. Clin Microbiol Rev 2007, 20(1):133-163.

27. Fridkin SK, Kaufman D, Edwards JR, Shetty S, Horan T: Changing incidence of Candida bloodstream infections among NICU patients in the United States: 1995-2004. Pediatrics 2006, 117(5):1680-1687.

28. Chang A, Neofytos D, Horn D: Candidemia in the 21 st century. Future Microbiol 2008, 3(4):463-472.

29. Shetty SS, Harrison LH, Hajjeh RA, Taylor T, Mirza SA, Schmidt AB, Sanza LT, Shutt KA, Fridkin SK: Determining risk factors for candidemia among newborn infants from population-based surveillance: Baltimore, Maryland, 1998-2000. Pediatr Infect Dis J 2005, 24(7):601-604.

30. Lerolle N, Trinquart L, Bornstain C, Tadié JM, Imbert A, Diehl JL, Fagon JY, Guérot E: Increased intensity of treatment and decreased mortality in elderly patients in an intensive care unit over a decade. Crit Care Med 2010, 38(1):59.

31. Halpern NA, Pastores SM: Critical care medicine in the United States 2000-2005: An analysis of bed numbers, occupancy rates, payer mix, and costs. Crit Care Med 2010, 38(1):65.

32. Giri S, Kindo AJ: A review of Candida species causing blood stream infection. Indian J Med Microbiol 2012, 30(3):270-278.

33. Ann Chai LY, Denning DW, Warn P: Candida tropicalis in human disease. Crit Rev Microbiol 2010, 36(4):282-298.

34. Wu J-Q, Zhu L-P, Ou X-T, Xu B, Hu X-P, Wang X, Weng X-H: Epidemiology and risk factors for non-Candida albicans candidemia in non-neutropenic patients at a Chinese teaching hospital. Medical Mycology 2011, 49(5):552-555.

35. Pfaller M, Diekema D, Gibbs D, Newell V, Bijie H, Dzierzanowska D, Klimko N, Letscher-Bru V, Lisalova M, Muehlethaler K: Results from the ARTEMIS DISK Global Antifungal Surveillance Study, 1997 to 2007: 10.5-year analysis of susceptibilities of noncandidal yeast species to fluconazole and voriconazole determined by CLSI standardized disk diffusion testing. J Clin Microbiol 2009, 47(1):117-123.

36. Peman J, Canton E, Gobernado M: Epidemiology and antifungal susceptibility of Candida species isolated from blood: results of a 2-year multicentre study in Spain. Eur J Clin Microbiol Infect Dis 2005, 24(1):23-30.

37. Ostrosky-Zeichner L, Lashof AO, Kullberg B, Rex J: Voriconazole salvage treatment of invasive candidiasis. Eur J Clin Microbiol Infect Dis 2003, 22(11):651-655.

38. Muñoz P, Sánchez-Somolinos M, Alcalá L, Rodríguez-Créixems M, Peláez T, Bouza E: Candida krusei fungaemia: antifungal susceptibility and clinical presentation of an uncommon entity during 15 years in a single general hospital. J Antimicrob Chemother 2005, 55(2):188-193.

39. Diekema D, Messer S, Brueggemann A, Coffman S, Doern G, Herwaldt L, Pfaller M: Epidemiology of candidemia: 3-year results from the emerging infections and the epidemiology of lowa organisms study. J Clin Microbiol 2002, 40(4):1298-1302.

40. Xess I, Jain N, Hasan F, Mandal P, Banerjee U: Epidemiology of candidemia in a tertiary care centre of north India: 5-year study. Infection 2007, 35(4):256-259. 
41. Quindós G, Abarca L, Carrillo-Muñoz AJ, Arévalo MP, Bornay FJ, Casals JB, Molina JMH, Iglesias I, Linares M, Martín-Mazuelos E: Multicenter survey of in vitro antifungal resistance in yeasts of medical importance isolated from Spanish patients. Rev Iberoam Micol 1999, 16:97-100.

42. Bassetti M, Righi E, Costa A, Fasce R, Molinari MP, Rosso R, Pallavicini FB, Viscoli C: Epidemiological trends in nosocomial candidemia in intensive care. BMC Infect Dis 2006, 6(1):21.

43. Bassetti M, Trecarichi EM, Righi E, Sanguinetti M, Bisio F, Posteraro B, Soro O, Cauda R, Viscoli C, Tumbarello M: Incidence, risk factors, and predictors of outcome of candidemia. Survey in 2 Italian university hospitals. Diagn Microbiol Infect Dis 2007, 58(3):325-331.

44. Al-Tawfiq JA: Distribution and epidemiology of Candida species causing fungemia at a Saudi Arabian hospital. Int I Infect Dis 2007, 11:239-244.

45. Costa S, Marinho I, Araujo E, Manrique A, Levin E: Nosocomial fungaemia: a 2-year prospective study. J Hosp Infect 2000, 45(1):69-72

46. Blumberg HM, Jarvis WR, Soucie JM, Edwards JE, Patterson JE, Pfaller MA, Rangel-Frausto MS, Rinaldi MG, Saiman L, Wiblin RT: Risk factors for candidal bloodstream infections in surgical intensive care unit patients: the NEMIS prospective multicenter study. Clin Infect Dis 2001, 33(2):177-186.

doi:10.1186/1471-2334-13-337

Cite this article as: Ma et al: Surveillance study of species distribution, antifungal susceptibility and mortality of nosocomial candidemia in a tertiary care hospital in China. BMC Infectious Diseases 2013 13:337.

\section{Submit your next manuscript to BioMed Central and take full advantage of:}

- Convenient online submission

- Thorough peer review

- No space constraints or color figure charges

- Immediate publication on acceptance

- Inclusion in PubMed, CAS, Scopus and Google Scholar

- Research which is freely available for redistribution 